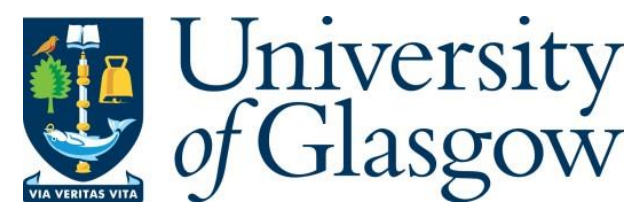

Akar, S., Rashidi, S., Esfahani, J.A. and Karimi, N. (2019) Targeting a channel coating by using magnetic field and magnetic nanofluids. Journal of Thermal Analysis and Calorimetry, 137(2), pp. 381-388. (doi:10.1007/s10973-018-7975-3)

There may be differences between this version and the published version. You are advised to consult the publisher's version if you wish to cite from it.

http://eprints.gla.ac.uk/174938/

Deposited on: 10 December 2018

Enlighten - Research publications by members of the University of Glasgow http://eprints.gla.ac.uk 


\title{
Targeting a channel coating by using magnetic field and magnetic nanofluids
}

\author{
S. Akar ${ }^{1}$, S. Rashidi $^{*}$, J.A. Esfahani $^{1}$, N. Karimi ${ }^{2}$ \\ ${ }^{1}$ Department of Mechanical Engineering, Ferdowsi University of Mashhad, Mashhad 91775-1111, Iran \\ ${ }^{2}$ School of Engineering, University of Glasgow, Glasgow G12 8QQ, UK \\ *Corresponding author: samanrashidi3983@gmail.com
}

\begin{abstract}
In this paper, the magnetic nanofluids and magnetic field are used to provide the coating around the wall of a channel. The magnetic field is induced by the direct current wire. Iron oxide is used as magnetic nanoparticles. A finite volume method is used to solve the Navier stokes equations and the Eulerian-Lagrangian approach is employed to track the magnetic nanoparticles. The effects of magnetic strength, the position of current wire, and the diameter of magnetic nanoparticles on the trajectory of magnetic nanoparticles and coating efficiency are investigated by providing contours are diagrams. The results showed that the length of coating decreases about $55 \%$ with increasing the particle diameter in the range of $500 \mathrm{~nm}$ to $1 \mu \mathrm{m}$. Further, the coating efficiency, defined as the ratio of the number of trapped particles on the wall to the number of injected particles at the inlet of the channel, improves by increasing the magnetic strength and decreasing the vertical position of current wire.
\end{abstract}

Keywords: Coating; Magnetic field; Magnetic nanoparticles; Finite volume method; EulerianLagrangian approach 


\section{Nomenclature}

$B \quad$ Magnetic field magnitude $(T)$

$B_{\max } \quad$ Maximum magnetic field magnitude $(T)$

$D \quad$ Width of channel $(m)$

$\mathrm{d}_{p} \quad$ Particle diameter $(m)$

$C_{D} \quad$ Drag coefficient (-)

F $\quad$ Total force on cell $(N)$

$\vec{H} \quad$ Magnetic field intensity $\left(A . m^{-1}\right)$

$I \quad$ Current (A)

$L \quad$ Length of channel (m)

$M_{\text {sat }} \quad$ Saturated magnetization of nanoparticles $\left(A \cdot m^{-1}\right)$

$\vec{M} \quad$ Magnetization $\left(A . m^{-1}\right)$

$N \quad$ Number of particles (-)

Re Reynolds number (-) $\operatorname{Re}=\frac{\rho_{\text {eff }} \mathrm{UD}}{\mu_{\text {eff }}}$

$\vec{U} \quad$ Average velocity $\left(m s^{-1}\right)$

$u \quad$ Velocity in $\mathrm{x}$ direction $\left(m s^{-1}\right)$

$\vec{V} \quad$ Velocity vector $\left(m s^{-1}\right)$

$V_{p} \quad$ Volume of particle $\left(\boldsymbol{m}^{3}\right)$

$\boldsymbol{v} \quad$ Velocity in y-direction $\left(m s^{-1}\right)$

$\mathrm{y}_{\text {mag }} \quad$ Vertical position of current wire

\section{Subscripts}




$\begin{array}{ll}\text { B } & \text { Brownian } \\ \text { D } & \text { Drag } \\ \text { F } & \text { Fluid } \\ \text { i } & \text { injected } \\ \text { M } & \text { Magnetic } \\ \text { P } & \text { Particle } \\ \text { t } & \text { trapped }\end{array}$

\section{Greek symbols}

$\begin{array}{ll}\eta & \text { Efficiency (-) } \\ \mu & \text { Dynamic viscosity }\left(\mathrm{kg} \cdot \mathrm{m}^{-1} \cdot \mathrm{s}^{-1}\right) \\ \mu_{0} & \text { Magnetic permeability of vacuum }\left(T \cdot m \cdot \mathrm{A}^{-1}\right) \\ \rho & \text { Density }\left(\mathrm{kg} \cdot \mathrm{m}^{-3}\right) \\ \chi & \text { Magnetic susceptibility }(-)\end{array}$

\section{Introduction}

Coatings play an important role in many technological areas including thermal industries, manufacturing of gas turbine engines widely used in aerospace industry, industrial power generations, color sciences, insulation industries, solar cells, and marine applications. Providing a proper coating can enhance the performance and useful life of the system [1-3]. Moreover, most of metals are working under varying harsh conditions and environments. For example, in different industrial processes, even flexible metals can be exposed to serious damage. In these situations, they should be protected by proper coatings. Researchers have used a number of 
techniques for coating including blade coating [4], spin coating [5], spray coating [6] and dip coating [7] to name few.

Aziz and Ismail [8] reviewed the printing and coating techniques. Their review showed that the spray coating technique has no limitation in coating layer thickness and low usage of polymers that is suitable to replace the conventional spin coating techniques. Jafari et al. [9] employed the plasma spraying technique to provide the composite coating along the surface of the 304stainless steel. They described this method as being very controllable and economical, which can be used to provide the coatings against the wear and corrosion. Sing et al. [10] used the dipcoating technique to provide the proper absorber layer for perovskite solar cell. They reported that a uniform coating can be achieved by using the optimized dipping, deposition, and evaporating parameters.

Recently, using nanofluids has attracted significant attentions especially in the field of thermal sciences and many studies used nanofluids to improve the heat transfer rate in different thermal systems [11-13]. A large part of the investigations performed in the field of nanofluid have been numerical studies. Several models are employed to simulate the heat transfer characteristics and flow structures of nanofluids in the recent years $[14,15]$. A promising and accurate model to simulate the flow and heat transfer of nanofluids is the discrete particle model $[16,17]$. In this model, the particles can be individually tracked employing a Lagrangian trajectory analysis technique, while the base-fluid can be modeled employing an Eulerian frame [18]. Rashadi et al. [19] employed the discrete particle model to simulate nanofluid flow and heat transfer across a triangular cylinder. They compared the results obtained by both discrete particle and singlephase models with the available experimental data. This comparison showed that the discrete particle model provides the results with more accuracy as compared with the effective single- 
phase model. They also found that the concentration of nanoparticle reduces as the nanoparticle size increases. In another numerical study, Bovand et al. [20] used Eulerian-Lagrangian model to simulate nanofluid flow and heat transfer inside a channel. They considered two particle boundary conditions, trap and reflect boundary conditions, at the walls of the channel. They concluded that for a reflecting surface, the concentration near the surface increases marginally which can lead to a slight diffusion away from the surface. However, for a trapping surface, a sharp concentration gradient around the surface occurred and the particles diffused toward the surface.

Recently, magnetic nanofluids and magnetohydrodynamics are widely used in different thermal systems due to their ability of controlling the flow and heat transfer process by a magnetic field [21 to 24]. Moreover, the thermo-physical properties of magnetic nanofluids can be controlled using an external magnetic field. Nkurikiyimfura et al. [25] and Bahiraei and Hangi [26] reviewed the studies performed in the field heat transfer in magnetic nanofluids. Nkurikiyimfura et al. [25] and Bahiraei and Hangi [26] concluded that the thermo-physical characteristics of magnetic nanofluids are heavily dependent upon the conditions of the imposed magnetic field including the strength and direction of the magnetic field. Besides the application of magnetic nanofluids in thermal systems, they may be used in biological systems. Rashidi et al. [27] reviewed the potentials of magnetohydrodynamics and magnetic nanofluids in different biological systems. These authors focused on the numerical studies performed in this field and showed that the external magnetic field can be utilized to deliver the particles towards a target region. Choomphon-anomakhun et al. [28] presented a model and simulated the dynamics of magnetic particles near a Ferromagnetic wire. In their model, Navier-Stokes equations are solved for fluid flow and the continuity equations are used separately for the base fluid and the magnetic 
particle phases. Moreover, a Laplace equation is considered for simulating the magnetic potential of the wire. Bernad et al. [29] conducted experimental and numerical work to study the effects of a magnetic field on the particles deposition in a coronary bypass graft. Their results indicated that particle deposition is related to the bypass graft angle.

In this numerical study, the magnetic field is used to provide the coating around the wall of a channel by Iron oxide-water nanofluids. The magnetic field can be induced by a direct current wire. An Eulerian-Lagrangian approach is employed to track the magnetic nanoparticles. The effects of magnetic strength, the position of current wire, and the diameter of magnetic nanoparticles on the trajectory of the magnetic nanoparticles in the base fluid and coating efficiency are investigated. This is for first time that this method is used for coating purposes.

\section{Physical model}

Figure1 shows the geometry of the computational domain. As shown in this figure, a channel with width $\mathrm{D}=0.01 \mathrm{~m}$ and length $\mathrm{L}=20 \mathrm{D}$ is considered. It is assumed that the $\mathrm{Fe}_{3} \mathrm{O}_{4}$-water nanofluid enters the channel with a uniform velocity. A current wire is located at vertical position of $y_{\text {mag }}$ to generate the magnetic field. The following assumptions are made throughout this study:

- The flow is steady, laminar, two-dimensional, incompressible, and Newtonian.

- The density and susceptibility of $\mathrm{Fe}_{3} \mathrm{O}_{4}$ nanoparticle are $5100 \mathrm{kgm}^{-3}$ and 2.1, respectively.

The physical properties of magnetic nanofluid are presented in Table 1.

$\underline{\text { Insert figure } 1 \text { here }}$

$\underline{\text { Insert Table } 1 \text { here }}$

\section{Mathematical model}


An Eulerian-Lagrangian method is employed to simulate the nanofluid flow inside the channel [30]. In this method, the Eulerian approach is considered to analyze the fluid flow, while the Lagrangian method is used to track the trajectories of magnetic particles. In this approach, the discrete phase magnetic particles are carried by the continuous phase. Indeed, the momentum can be exchanged between the particles and base fluid by the drag force between them [30]. The governing equations of the two phases are presented as follows.

\subsection{Governing equation}

\subsubsection{Continuum phase}

For the continuum phase (water), the following equations are used:

Mass conservation equation:

$\vec{W}=0$

Momentum equation:

$\vec{V} \cdot \vec{W}=-\vec{\nabla} \cdot p+\frac{1}{\operatorname{Re}} \nabla^{2} \vec{V}$

where $\vec{V}$, p, and Re are velocity vector, pressure, and Reynolds number, respectively.

\subsubsection{The dispersed phase}

Motions of the magnetic nanoparticles or dispersed phase are simulated employing the Lagrangian particle trajectory approach. Hence, the particle inertia is balanced with the other forces exerting on the particles. The equation of motion for the magnetic nanoparticles is presented as follows:

$m_{c} \frac{d u_{c}}{d t}=\vec{F}$

where parameter $\vec{F}$ is the total force acted on the magnetic defined by, 
$\vec{F}=\overrightarrow{F_{D}}+\overrightarrow{F_{M}}+\overrightarrow{F_{B}}$

where $\overrightarrow{F_{D}}, \overrightarrow{F_{M}}$, and $\overrightarrow{F_{B}}$ are drag, magnetic, and Brownian forces, respectively.

The Stokes drag force on a spherical particle presented by Morsi and Alexander [31] is used in this study. This force is calculated by

$F_{D}=\frac{18 \mu_{f} C_{D} \operatorname{Re}}{24 \rho_{p} d_{p}^{2}}$

where $d_{p}, \rho_{p}$, and $C_{D}$ indicate nanoparticle diameter, nanoparticle density, and the drag coefficient respectively. The drag coefficient can be calculated by

$C_{D}=a_{1}+\frac{a_{2}}{\operatorname{Re}}+\frac{a_{3}}{\operatorname{Re}^{2}}$

where $a_{1}, a_{2}, a_{3}$ are constants.

The magnetic force is defined as [32],

$F_{m a g}=V_{p} \mu_{0} M \nabla H$

where $\mu_{0}, V_{P}, \mathrm{M}$, and $\mathrm{H}$ denote permeability of vacuum, volume of magnetic nanoparticles, magnetization of magnetic nanoparticle, and magnetic field intensity, respectively. The magnetization of the magnetic nanoparticle can be calculated using the following expression [32]:

$\vec{M}= \begin{cases}\psi \vec{H} & \text { for } H<M s a t / \psi \\ M_{\text {sat }} \widehat{\mathrm{H}} & \text { for } H>M s a t / \psi\end{cases}$

where $M_{\text {sat }}$ and $\psi$ denote the magnetism saturation and magnetic nanoparticle susceptibility, respectively. The magnetic field intensity, $\mathrm{H}$, created by wire carrying the current of $\mathrm{I}$ is calculated by,

$H=\frac{I}{2 \pi\left(y-y_{\text {mag }}\right)}$ 
where $y_{\text {mag }}$ denotes the vertical position of the current wire.

\subsection{Boundary conditions}

\subsubsection{Continuum phase}

At the inlet section of the channel the following boundary condition is used for the continuum phase:

$u=u_{\text {in }}, \mathrm{v}=0$

where $\mathrm{u}$ and $\mathrm{v}$ are the horizontal and vertical velocities, respectively.

At the outlet section of the channel, the zero gradient boundary condition is used as follows:

$\partial u / \partial x=0$

At the walls of the channel, the following no-slip boundary condition is used:

$u=0, \mathrm{v}=0$

\subsubsection{The dispersed phase}

At the inlet and outlet of the channel, the escape boundary condition is used for the dispersed phase (magnetic nanoparticles). This indicates that the trajectory calculations are terminated for particles after leaving the channel. Moreover, the trap boundary condition with the coefficient of restitution equal to 0 is used at the walls of the channel. Note that it is assumed that the trajectory calculations for the particles are terminated on the walls by using the trap boundary condition.

\subsection{Coating efficiency}

The coating efficiency is defined as follows:

$\eta=\frac{N_{t}}{N_{i}}$

where $\mathrm{N}_{t}$ and $\mathrm{N}_{\mathrm{i}}$ are the number of trapped particles on the wall and the number of injected particles at the inlet of the channel, respectively. 


\section{Numerical solution}

A pressure base finite volume method is used to solve the governing equations for the continuous phase, equations 1 and 2, with their boundary conditions. A staggered computational grid is used to store the pressure and velocity terms at cell center and cell faces, respectively. SIMPLE approach [33] is employed to couple pressure and velocity terms. A second-order upwind differencing method is utilized to discretize the equations. For dispersed phase, the modified Euler technique is employed to discretize the particle equation. Finally, the converged solution criteria are passed as the summation of residuals to be smaller than $10^{-7}$ for all equations. All simulations are carried out by the commercial software Ansys-Fluent.

\subsection{Grid independency study}

The typical grid generated inside the computational domain is shown in Fig. 2. As shown in this figure, a square mesh is used in this study. This mesh is fined near the channel walls, where there are large gradients. A grid independency study is conducted here to ensure that the results are independent of the grid size. Table 2 presents the effects of grid number on coating efficiency for $\operatorname{Re}=100, \mathrm{y}_{\mathrm{mag}}=2.5 \mathrm{~cm}$ and $\mathrm{dp}=10^{-7}$. It can be seen that the percentage difference of coating efficiency for grid numbers of $160 \times 1000$ and $320 \times 2000$ is only $0.4 \%$, which is negligible. Accordingly, the grid number of $160 \times 1000$ is selected for the rest of simulations presented in this study.

\section{Insert figure 2 here}

\section{$\underline{\text { Insert Table } 2 \text { here }}$}

\subsection{Validation}

To validate the numerical solver, the particle trajectory in the microchannel in the presence of the magnetic field is determined and compared with the analytical results presented by Nandi et al. 
[34]. The dimensions of the simulated microchannel is $150 \mu \mathrm{m} \times 7.5 \mathrm{~mm}$ and a magnetic dipole with strength of $1.98 \times 10^{-6}$ and position of $x=3.72 \times 10^{-3} \mu \mathrm{m}$ and $y=-3 \times 10^{-5} \mu \mathrm{m}$ from the origin is exerted. The magnetic particle with diameter of $2 \mu \mathrm{m}$ is inserted. Figure 3 compared the trajectories of the magnetic particles inside this microchannel in the presence of magnetic field. As shown in this figure, a good agreement between the two results can be observed.

\section{Insert figure 3 here}

\section{Result and discussion}

The results of numerical simulations are presented in this section for magnetic strengths in the range of 1 to 2 Tesla, the vertical positions of magnetic dipole in the range of $1.25 \mathrm{D}$ to $3.75 \mathrm{D}$, and the diameters of magnetic nanoparticles in the range of 500 to $1000 \mathrm{~nm}$. All simulations are performed for a fixed value of Reynolds number at $\operatorname{Re}=100$. The effects of magnetic strength, the position of current wire, and the diameter of magnetic nanoparticles on the trajectory of magnetic nanoparticles and coating efficiency are investigated by providing contours and diagrams.

Figure 4 plots the contour of magnetic field at $R e=100, B_{\max }=1.5$ Tesla and $y_{\operatorname{mag}}=2.5 \mathrm{~cm}$. As shown in this figure, the magnetic field is stronger near the top wall of the channel as the wire is located near this wall. Note that in this study, the channel is exposed to a non-uniform transverse magnetic field produced by an electric current going through a wire placed in parallel and at the vertical distance of $\mathrm{y}_{\mathrm{mag}}$ from the coordinate system. Based on Eq. 9, there is an indirect relationship between the strength of magnetic field at a point and the vertical distance of wire from that point.

\section{Insert figure 4 here}

Figure 5 shows the effects of magnetic field on the particle trajectory at $\operatorname{Re}=100, \mathrm{y}_{\mathrm{mag}}=2.5 \mathrm{~cm}$, $B_{\max }=1.5$ Tesla, and $d_{p}=500 \mathrm{~nm}$. As shown in this figure, for the case of without magnetic field, 
the particles are moving through the duct without any deviation from the flow streamlines. However, the particles are deviated towards the upper wall by exerting the magnetic field. The particles are affected by the magnetic force generated by the magnetic field. This force acts on the particle and the particles are deviated towards the upper wall of the channel by the vertical component of this force. As a result, a coated layer of particle can be generated on the upper wall of the channel by exerting the magnetic field.

\section{Insert figure 5 here}

The effects of magnetic particle diameter on particle trajectory at $\mathrm{Re}=100, \mathrm{ymag}_{\mathrm{m}}=2.5 \mathrm{~cm}$, and $\mathrm{B}_{\max }=1.5$ Tesla are disclosed in Fig. 6. It should be mentioned that with increasing the diameter of nanoparticles, the nanoparticle volume increases by a factor of three, resulting in a large magnetic force (see Eq. 9). Accordingly, particles are affected by a larger magnetic force, resulting in the higher sediment yields. This figure shows that larger particles are absorbed by the upper wall in a shorter distance from the inlet as compared with the smaller particles. This indicates that it is possible to provide a local coating only at the entrance regions of the channel by using particles with larger diameters. It is worth mentioning that the color of the particle trajectory in the contours also indicates the strength of the magnetic field with the unit of Tesla.

\section{Insert figure 6 here}

The effects of strength of the magnetic field on the particle trajectory at $\mathrm{Re}=100, \mathrm{y}_{\mathrm{mag}}=2.5 \mathrm{~cm}$, and $d_{p}=300 \mathrm{~nm}$ are shown in Fig. 7. This figure shows that as the strength of the magnetic field increases for the particles with same diameter, more nanoparticles are absorbed by the upper wall, which results in better coating. Note that the magnetic force is strengthened with increasing the magnetic field intensity and this increases the power of the wire to absorb nanoparticles.

\section{Insert figure 7 here}


Variations of coating efficiency with magnetic nanoparticles diameter for three magnetic field strengths at $\mathrm{Re}=100$ and $\mathrm{y}_{\mathrm{mag}}=2.5 \mathrm{~cm}$ are shown in Fig. 8 . As implied by this figure, the coating efficiency enhances with increasing the magnetic nanoparticles diameter for all magnetic field strengths. The coating efficiency improves by about $1020 \%, 900 \%$, and $540.1 \%$ as the magnetic nanoparticles diameter increases in the range of 0.1 to $0.7 \mu \mathrm{m}$ for the magnetic field strengths of $1,1.5$, and 2 Tesla, respectively. Note that, the drag force increases with increasing the particles diameter, which has a effect on the coating efficiency. However, the magnetic force enhances as the particles diameter increases, leaving a which has positive effect on the coating efficiency. With considering both forces and the fact that the magnetic force has a dominant effect on the coating efficiency, a better coating can be achieved by using larger particles. Moreover, the coating efficiency enhances with increasing the magnetic field strength. A larger magnetic force can be generated by exerting a magnetic field with higher strength. The coating efficiency enhances by about $1900 \%$ with increasing the magnetic field strength in the range of 1 to 2 Tesla for the magnetic nanoparticles with diameter of $0.1 \mu \mathrm{m}$. However, for magnetic nanoparticles with diameter of $0.7 \mu \mathrm{m}$, the coating efficiency does not change as the magnetic field strength increases from 1.5 to 2 Tesla.

\section{Insert figure 8 here}

The effects of wire positions on the particle trajectory at $\mathrm{Re}=100, \mathrm{~B}_{\max }=1.5$ Tesla, and $\mathrm{d}_{\mathrm{p}}=300$ $\mathrm{nm}$ are illustrated in Fig. 9. As shown in this figure, if the vertical distance between the wire and the channel decreases, the coating efficiency increases, and the particles are absorbed by the upper wall in a shorter distance from the inlet of channel. As mentioned earlier, the magnetic field intensity induced by the wire increases as the vertical distance between the wire and the 
channel decreases. This leads to generation of a stronger magnetic force, which results in a local coating at the entrance regions of the channel.

\section{Insert figure 9 here}

Variations of coating efficiency with magnetic nanoparticles diameter for three wire positions at $\operatorname{Re}=100$ and $B_{\max }=1.5$ Tesla are disclosed in Fig. 10. It can be seen that the coating efficiency enhances with increasing the magnetic nanoparticles diameter for the wire positions of $2.5 \mathrm{D}$ and 3.75D. The coating efficiency improves by $764.30 \%$ and $2072.72 \%$ as the magnetic nanoparticles diameter increases in the range of 0.1 to $0.7 \mu \mathrm{m}$ for the wire positions of $2.5 \mathrm{D}$ and 3.75D, respectively. However, for the wire position of $1.25 \mathrm{D}$, the coating efficiency does not change with increasing the magnetic nanoparticles diameter for $d_{p}>0.2 \mu \mathrm{m}$. In addition, the coating efficiency enhances with decreasing the vertical position of the wire. The coating efficiency enhances by $2969.76 \%$ with decreasing the vertical position of the wire in the range of $3.75 \mathrm{D}$ to $1.25 \mathrm{D}$ for the magnetic nanoparticles with diameter of $0.1 \mu \mathrm{m}$. However, for the magnetic nanoparticles with diameter of $0.7 \mu \mathrm{m}$, the coating efficiency does not change as the vertical position of the wire decreases from $2.5 \mathrm{D}$ to $1.25 \mathrm{D}$.

\section{Insert figure 10 here}

\section{Conclusions}

In this paper, a numerical simulation was performed to evaluate the potentials of the magnetic nanoparticles and magnetic field to provide the coating around the wall of a channel. The effects of different parameters including the magnetic strength, the position of current wire, and the diameter of magnetic nanoparticles on the trajectory of magnetic nanoparticles and coating efficiency are investigated by providing contour plots. The following conclusions are made from this study: 
- The length of coating decreases about $55 \%$ with increasing the particle diameter in the range of $500 \mathrm{~nm}$ to $1 \mu \mathrm{m}$.

- As the strength of the magnetic field increases for the particles with the same diameter, more nanoparticles are absorbed by the upper wall, which results in better coating.

- The coating efficiency improves by about $1020 \%, 900 \%$, and $540.1 \%$ as the magnetic nanoparticles diameter increases in the range of 0.1 to $0.7 \mu \mathrm{m}$ for the magnetic field strengths of $1,1.5$, and 2 Tesla, respectively.

- The coating efficiency enhances by $2969.72 \%$ with decreasing the vertical position of the wire in the range of $3.75 \mathrm{D}$ to $1.25 \mathrm{D}$ for the magnetic nanoparticles with diameter of $0.1 \mu \mathrm{m}$.

- The coating efficiency enhances by about $1900 \%$ with increasing the magnetic field strength in the range of 1 to 2 Tesla for the magnetic nanoparticles with diameter of $0.1 \mu \mathrm{m}$.

\section{References}

[1] K. Mydłowska, P. Myśliński, Ł. Szparaga, A. Gilewicz, J. Ratajski, “Analysis of the effect of antiwear $\mathrm{CrN}$ coating thickness on the evolution of thermomechanical interactions in the substrate/PVD coating system.' Journal of Thermal Analysis and Calorimetry, 125 (2016) $1241-1247$.

[2] A. Eslami, S.G. Hosseini, M. Bazrgary, "'Improvement of thermal decomposition properties of ammonium perchlorate particles using some polymer coating agents." Journal of Thermal Analysis and Calorimetry, 113 (2013) 721-730.

[3] Y. Sawada, S. Seki, M. Sano, N. Miyabayashi, K. Ninomiya, A. Iwasawa, T. Tsugoshi, R. Ozao, Y. Nishimoto, “Evolution of water vapor from indium-tin-oxide transparent conducting 
films fabricated by dip coating process." Journal of Thermal Analysis and Calorimetry, 77 (2004) 751-757.

[4] S. L. Lim, E. C. Chen, C. Y. Chen, K. H. Ong, Z. K. Chen, H. F. Meng, "'High performance organic photovoltaic cells with blade-coated active layers." Solar Energy Materials and Solar Cells, 107 (2012) 292-297.

[5] S.K. Jang, S.C. Gong, H.J. Chang, "Effects of various solvent addition on crystal and electrical properties of organic solar cells with P3HT:PCBM active layer.' Synthetic Metals, $162(5-6)(2012)$ 426-430.

[6] J.H. Lee, T. Sagawa, S. Yoshikawa, "Morphological and topographical characterizations in spray coated organic solar cells using an additional solvent spray deposition." Organic Electronics, 12 (12) (2011) 2165-2173.

[7] Z. Hu, J. Zhang, S. Xiong, Y. Zhao, “Performance of polymer solar cells fabricated by dip coating process." Solar Energy Materials and Solar Cells, 99 (2012) 221-225.

[8] F. Aziz, A.F. Ismail, "Spray coating methods for polymer solar cells fabrication: A review." Materials Science in Semiconductor Processing, 39 (2015) 416-425.

[9] M. Jafari, J. Mostaghimi, H. Monajatizadeh, M. Rafiei, "Microstructure evaluation of CO222/SiC coating produced by the plasma spraying method." Surface Engineering, 34 (2018) 220-225.

[10] R. Singh, I.S. Noor, Pramod K. Singh, B. Bhattacharya, A.K. Arof, "Synthesis of active absorber layer by dip-coating method for Perovskite solar cell." Journal of Molecular Structure, 1158 (2018) 229-233.

[11] S. Kakaç, A. Pramuanjaroenkij, "Review of convective heat transfer enhancement with nanofluids." International Journal of Heat and Mass Transfer, 52 (2009) 3187-3196. 
[12] L.S. Sundar, M.K. Singh, "Convective heat transfer and friction factor correlations of nanofluid in a tube and with inserts: a review." Renewable \& Sustainable Energy Reviews, 20 (2013) 23-35.

[13] S. Rashidi, O. Mahian, E.M. Languri, "Applications of nanofluids in condensing and evaporating systems." Journal of Thermal Analysis and Calorimetry, 131 (2018) 2027-2039.

[14] M. Sheikholeslami, H.B. Rokni, "Simulation of nanofluid heat transfer in presence of magnetic field: A review.' International Journal of Heat and Mass Transfer, 115 (2017) 12031233.

[15] N.A.C. Sidik, R. Mamat, "Recent progress on lattice Boltzmann simulation of nanofluids: A review." International Communications in Heat and Mass Transfer, 66 (2015) 11-22.

[16] M. Maskaniyan, S. Rashidi, J.A. Esfahani, "A two-way couple of Eulerian-Lagrangian model for particle transport with different sizes in an obstructed channel.' Powder Technology, 312 (2017) 260-269.

[17] S. Rashidi, J.A. Esfahani, R. Ellahi, "Convective heat transfer and particle motion in an obstructed duct with two side by side obstacles by means of DPM model." Applied Sciences, 7 (2017) 1-14.

[18] H. Afshar, M. Shams, S.M.M. Nainian, G. Ahmadi, "Microchannel heat transfer and dispersion of nanoparticles in slip flow regime with constant heat flux." International Communications in Heat and Mass Transfer, 36 (2009) 1060-1066.

[19] S. Rashidi, M. Bovand, J.A. Esfahani, G. Ahmadi, "Discrete particle model for convective $\mathrm{Al}_{2} \mathrm{O}_{3}$-water nanofluid around a triangular obstacle.” Applied Thermal Engineering, 100 (2016) $39-54$. 
[20] M. Bovand, S. Rashidi, G. Ahmadi, J.A. Esfahani, "Effects of trap and reflect particle boundary conditions on particle transport and convective heat transfer for duct flow-A two-way coupling of Eulerian-Lagrangian Model.” Applied Thermal Engineering, 108 (2016) 368-377.

[21] A. Majeed, A. Zeeshan, S.Z. Alamri, R. Ellahi, "Heat transfer analysis in ferromagnetic viscoelastic fluid flow over a stretching sheet with suction." Neural Computing and Applications, 30 (2018) 1947-1955.

[22] A. Zeeshan, N. Ijaz, T. Abbas, R. Ellahi, “The sustainable characteristic of Bio-bi-phase flow of peristaltic transport of MHD Jeffery fluid in human body." Sustainability, 10 (2018) 117.

[23] N. Shehzad, A. Zeeshan, R. Ellahi, "Electroosmotic flow of MHD Power law $\mathrm{Al}_{2} \mathrm{O}_{3}-\mathrm{PVC}$ nanofluid in a horizontal channel: Couette-Poiseuille flow model." Communications in Theoretical Physics, 69 (2018) 655-666.

[24] M .Hassan, M. Marin, A. Alsharif, R. Ellahi, "Convection heat transfer flow of nanofluid in a porous medium over wavy surface." Physics Letters A, 382 (2018) 2749-2753.

[25] A. Wakif, Z. Boulahia, S.R. Mishra, M.M. Rashidi, R. Sehaqui, “Influence of a uniform transverse magnetic field on the thermo-hydrodynamic stability in water-based nanofluids with metallic nanoparticles using the generalized Buongiorno's mathematical model.' The European Physical Journal Plus, 131 (2013) 181.

[26] A. Abdollahi, M.R. Salimpour, "Experimental investigation on the boiling heat transfer of nanofluids on a flat plate in the presence of a magnetic field." The European Physical Journal Plus, 131 (2016) 414. 
[27] S. Rashidi, J.A. Esfahani, M. Maskaniyan, "Applications of magnetohydrodynamics in biological systems-a review on the numerical studies." Journal of Magnetism and Magnetic Materials, 439 (2017) 358-372.

[28] N. Choomphon-anomakhun, A.D. Ebner, M. Natenapit, J.A. Ritter, "Simulation of dynamic magnetic particle capture and accumulation around a ferromagnetic wire." Journal of Magnetism and Magnetic Materials, 458 (2017) 493-505.

[29] S.I. Bernad, A.F. Totorean, L. Vekas, "Particles deposition induced by the magnetic field in the coronary bypass graft model."' Journal of Magnetism and Magnetic Materials, 401 (2016) 269-286.

[30] M. Mirzaei, M. Saffar-Avval, H. Naderan, "Heat transfer investigation of laminar developing flow of nanofluids in a microchannel based on Eulerian-Lagrangian approach." The Canadian Journal of Chemical Engineering, 92 (6) (2014) 1139-1149.

[31] S. Morsi, A. Alexander, "An investigation of particle trajectories in two-phase flow systems." Journal of Fluid Mechanics, 55 (1972) 193-208.

[32] M. Larimi, A. Ramiar, A. Ranjbar, "Numerical simulation of magnetic nanoparticles targeting in a bifurcation vessel." Journal of Magnetism and Magnetic Materials, 362 (2014) 5871.

[33] S.V. Patankar, Numerical heat transfer and fluid flow, (1980), Hemisphere, New York.

[34] K. Nandy, S. Chaudhuri, R. Ganguly, I.K. Puri, “Analytical model for the magnetophoretic capture of magnetic microspheres in microfluidic devices." Journal of Magnetism and Magnetic Materials, 320 (2008) 1398-1405. 


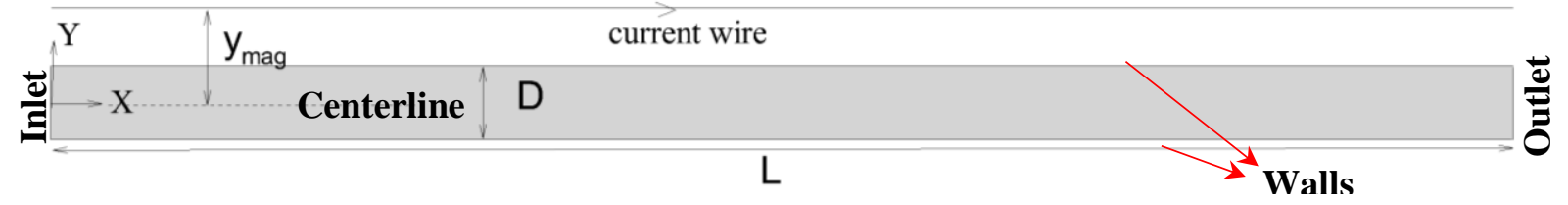

Fig.1. Schemitcis of the computational domain 


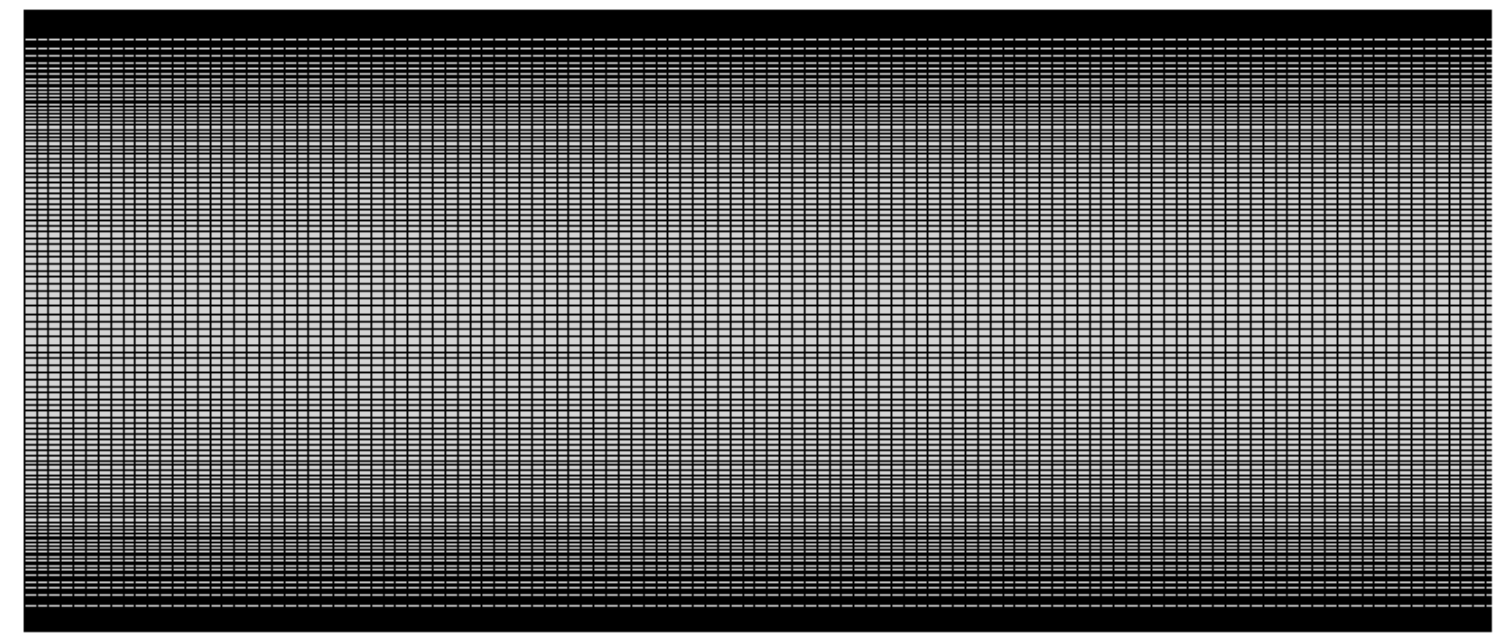

Fig. 2. Typical grid generated inside the domain 


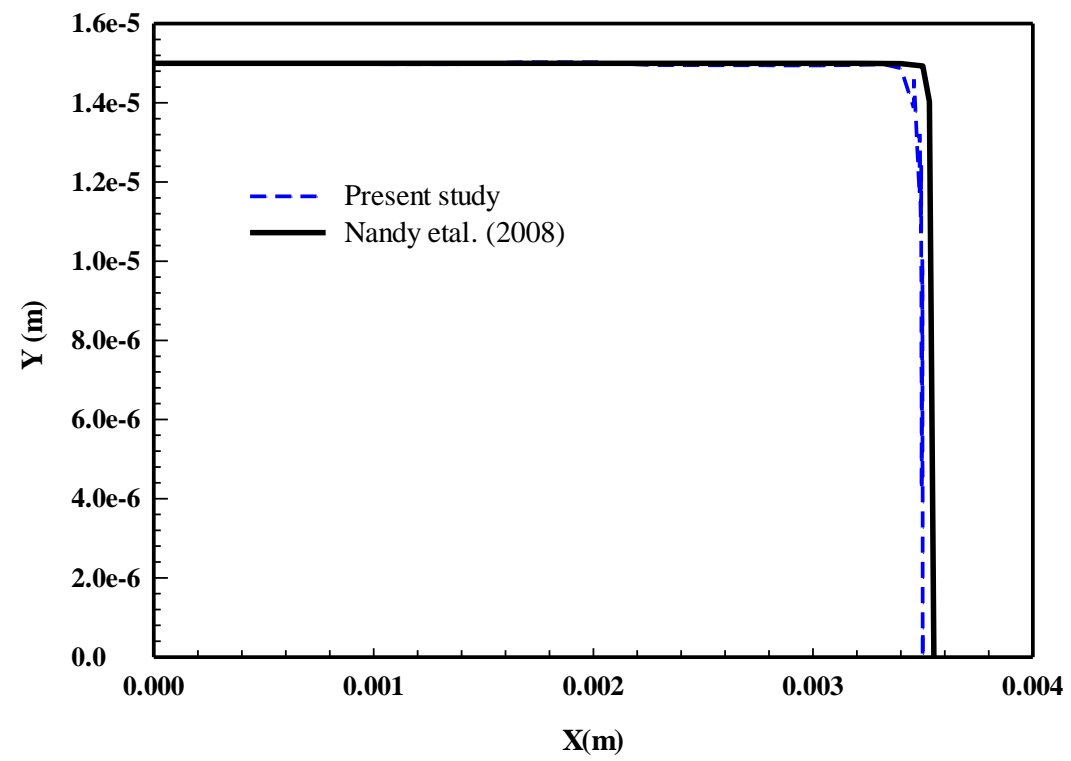

Fig. 3. Trajectories of the magnetic particles inside the microchannel in the presence of magnetic field. 
Magnetic field (Tesla)

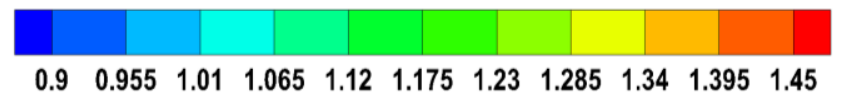

Fig.4. Contours of magnetic field at $B_{\max }=1.5$ Tesla and $y_{\operatorname{mag}}=2.5 \mathrm{~cm}$. 


\section{Without magnetic field}

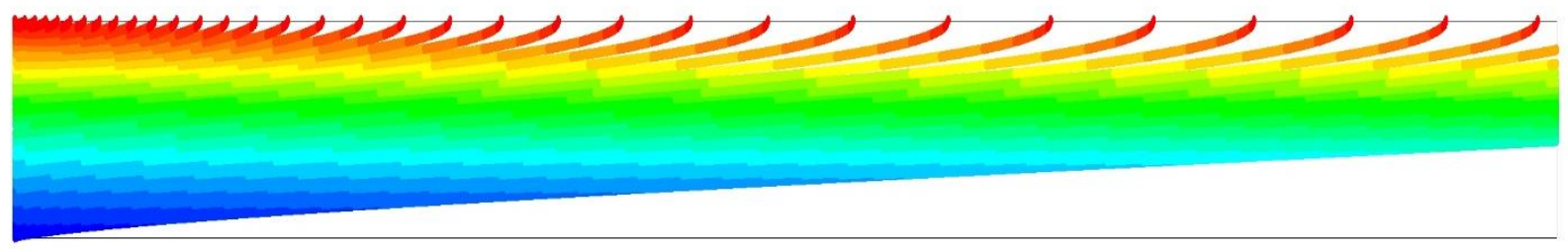

\section{With magnetic field}

Fig. 5. Effects of magnetic field on the particle trajectory at $R e=100, y_{\operatorname{mag}}=2.5 \mathrm{~cm}, B_{\max }=1.5$

Tesla, and $d_{p}=500 \mathrm{~nm}$. 

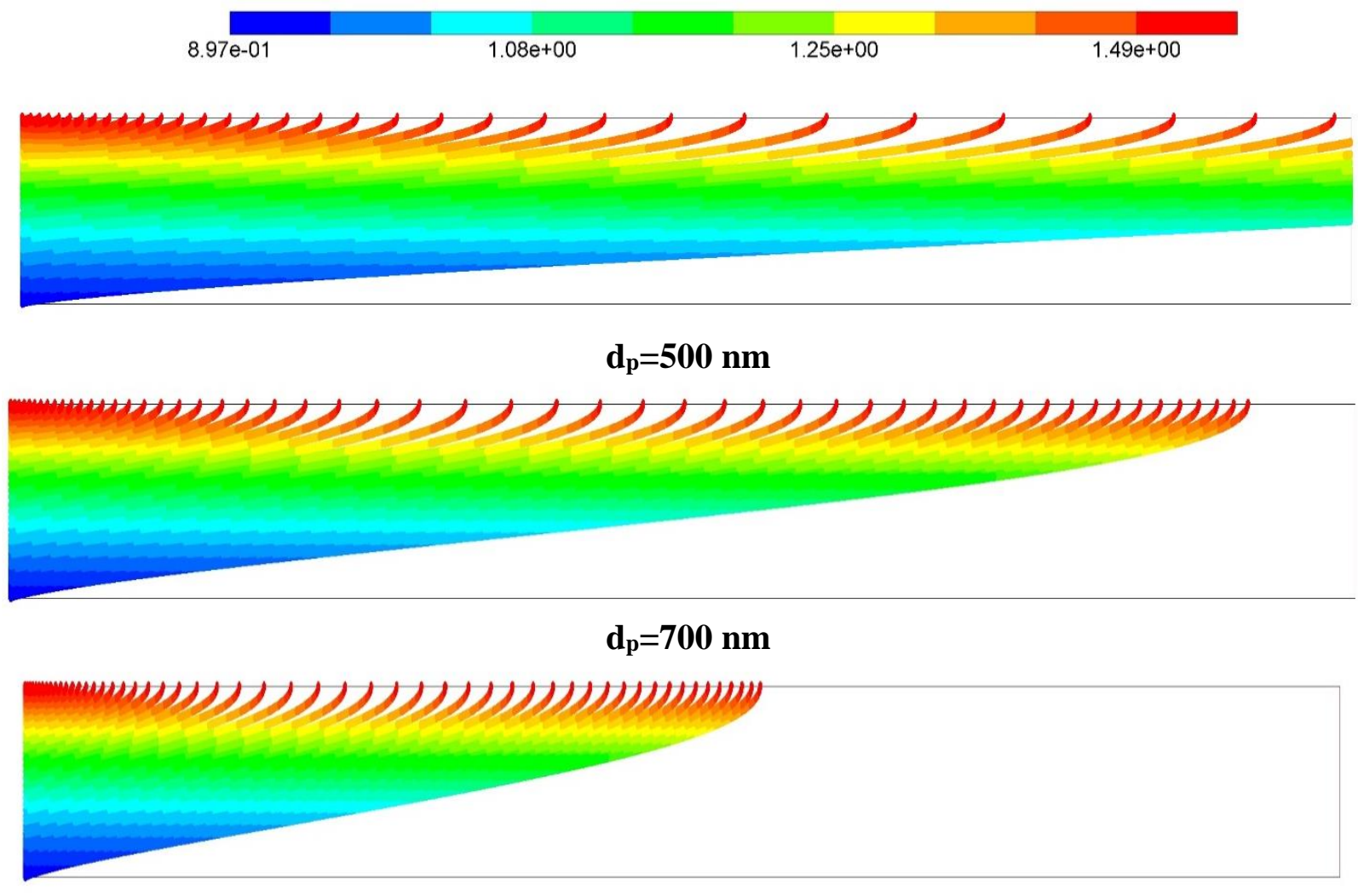

$$
d_{p}=900 \mathrm{~nm}
$$

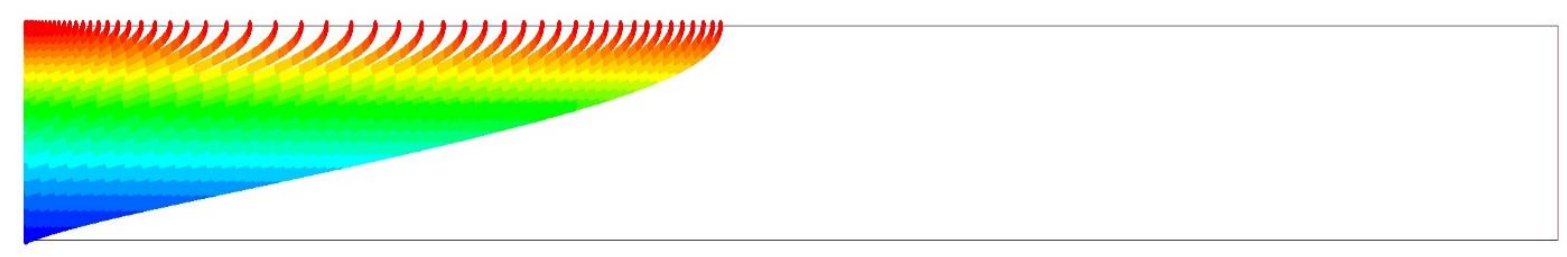

$$
\mathbf{d}_{\mathbf{p}}=\mathbf{1} \boldsymbol{\mu m}
$$

Fig. 6. Effects of magnetic particle diameter on the particle trajectory at $R e=100, y_{\mathrm{mag}}=2.5 \mathrm{~cm}$, and $\mathrm{B}_{\max }=1.5$ Tesla. 

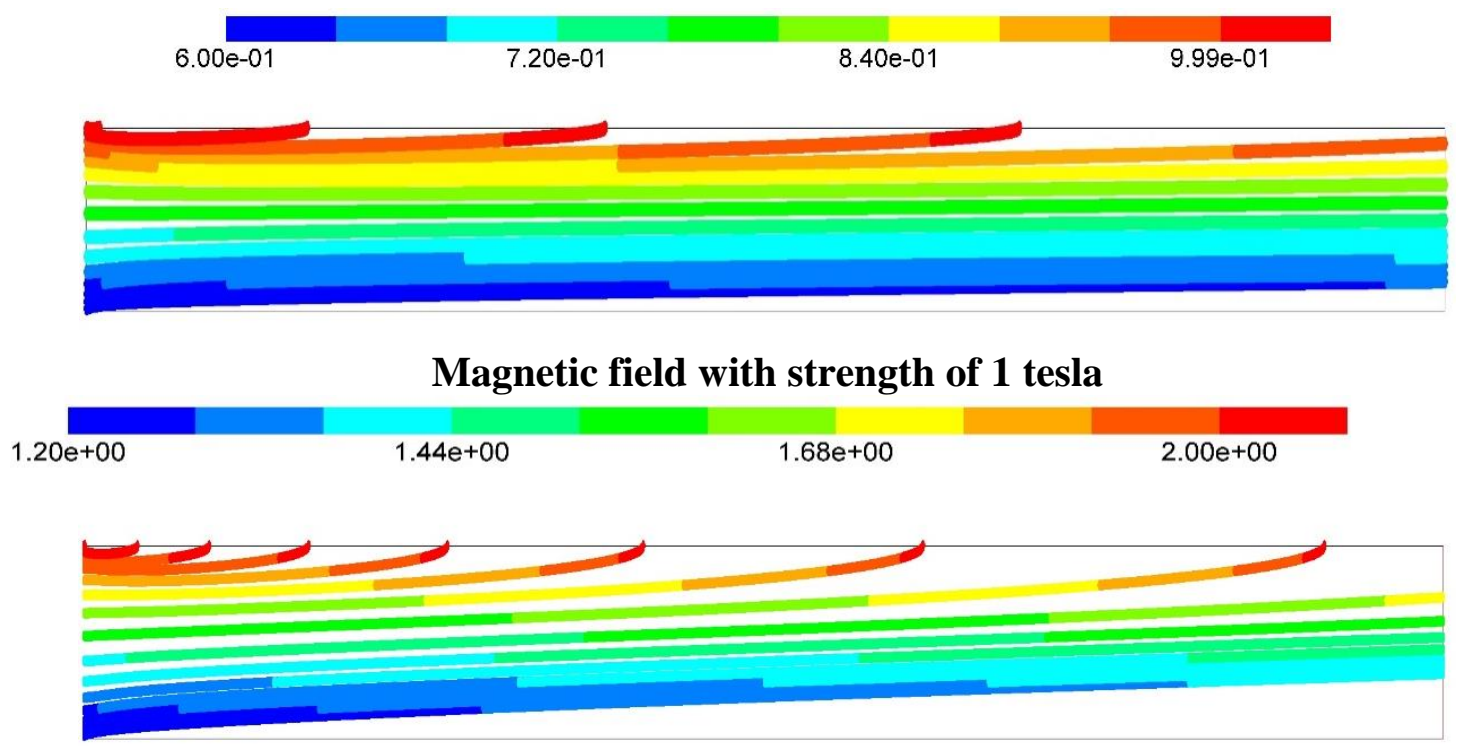

Magnetic field with strength of 2 tesla

Fig. 7. Effects of magnetic field strength on the particle trajectory at $\mathrm{Re}=100, \mathrm{ymag}_{\mathrm{ma}}=2.5 \mathrm{~cm}$ and

$$
\mathrm{d}_{\mathrm{p}}=300 \mathrm{~nm} \text {. }
$$




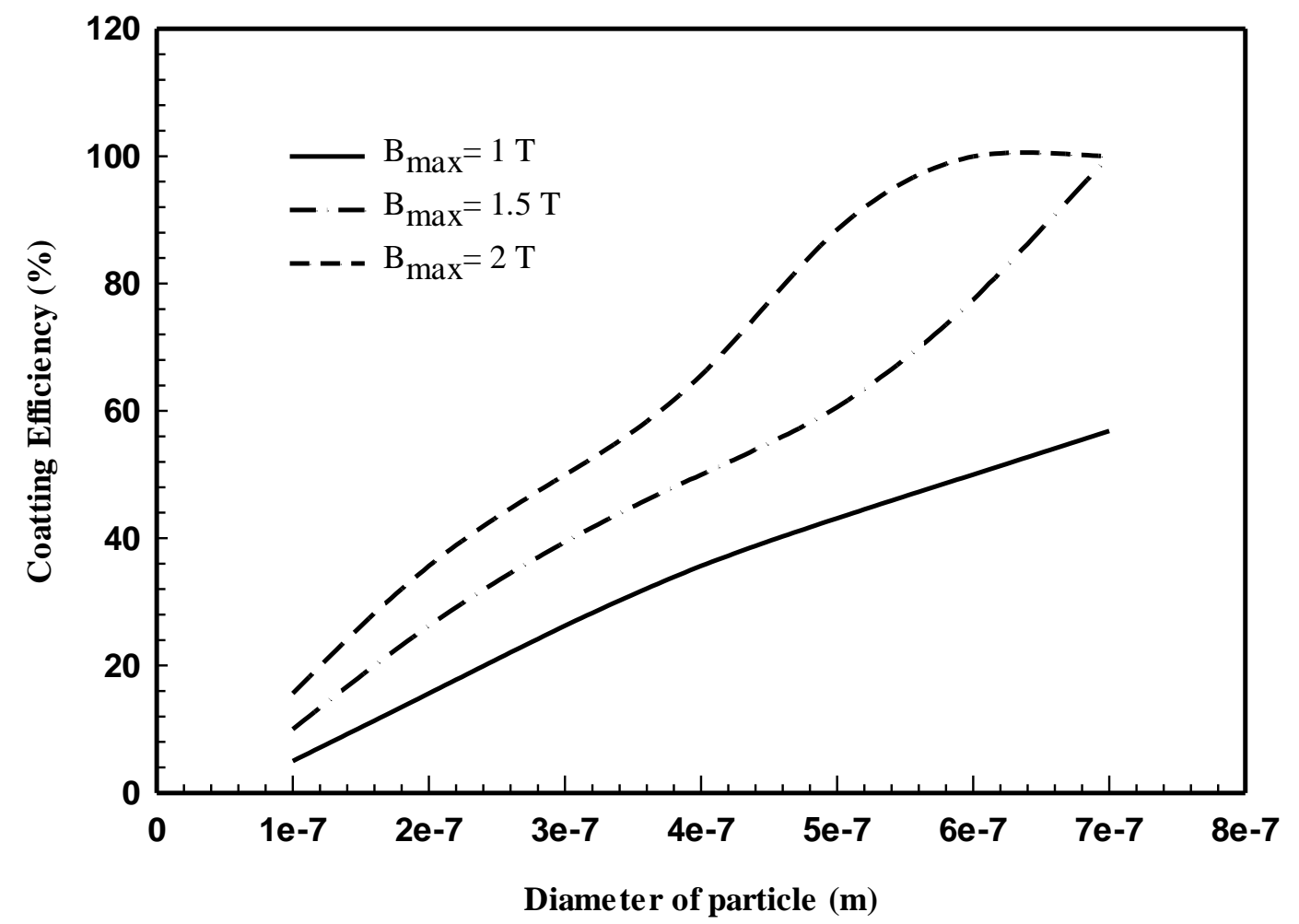

Fig. 8. Variations of coating efficiency with magnetic nanoparticles diameter for three magnetic field strengths at $\mathrm{Re}=100$ and $\mathrm{y}_{\mathrm{mag}}=2.5 \mathrm{~cm}$. 

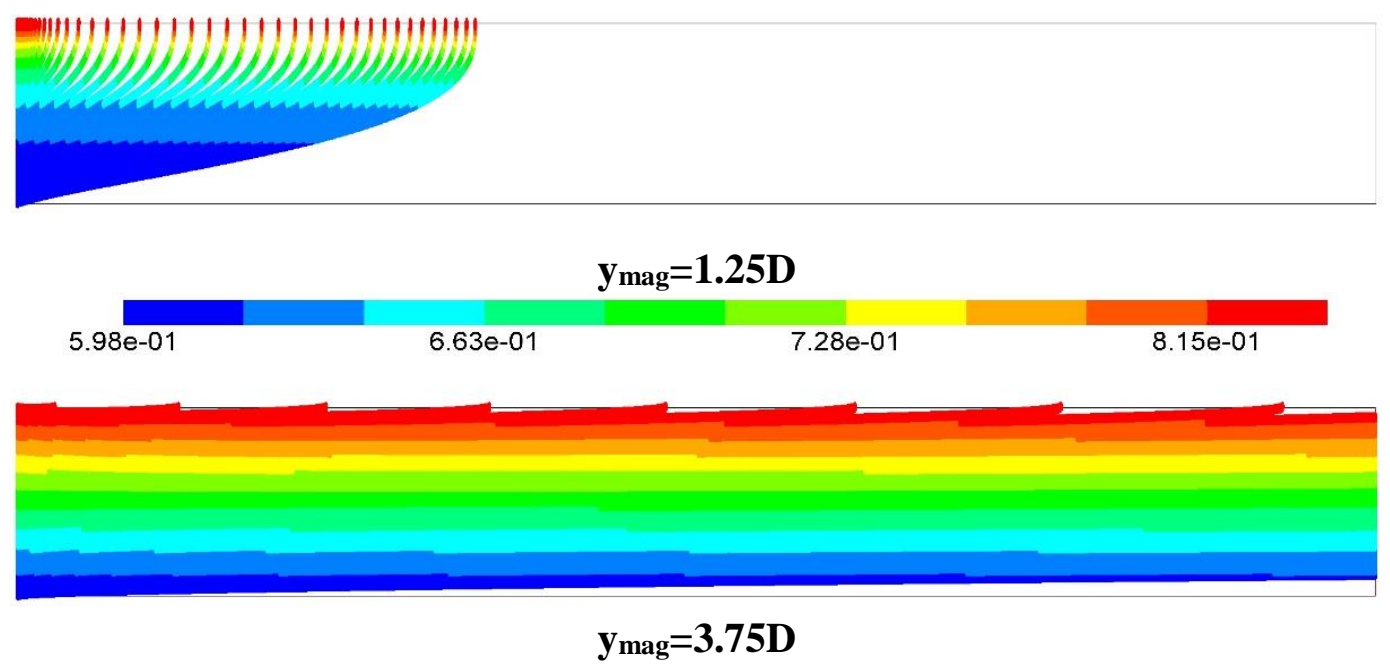

Fig. 9. Effects of wire positions on the particle trajectory at $R e=100, B_{\max }=1.5$ Tesla, and $d_{p}=300$

nm. 


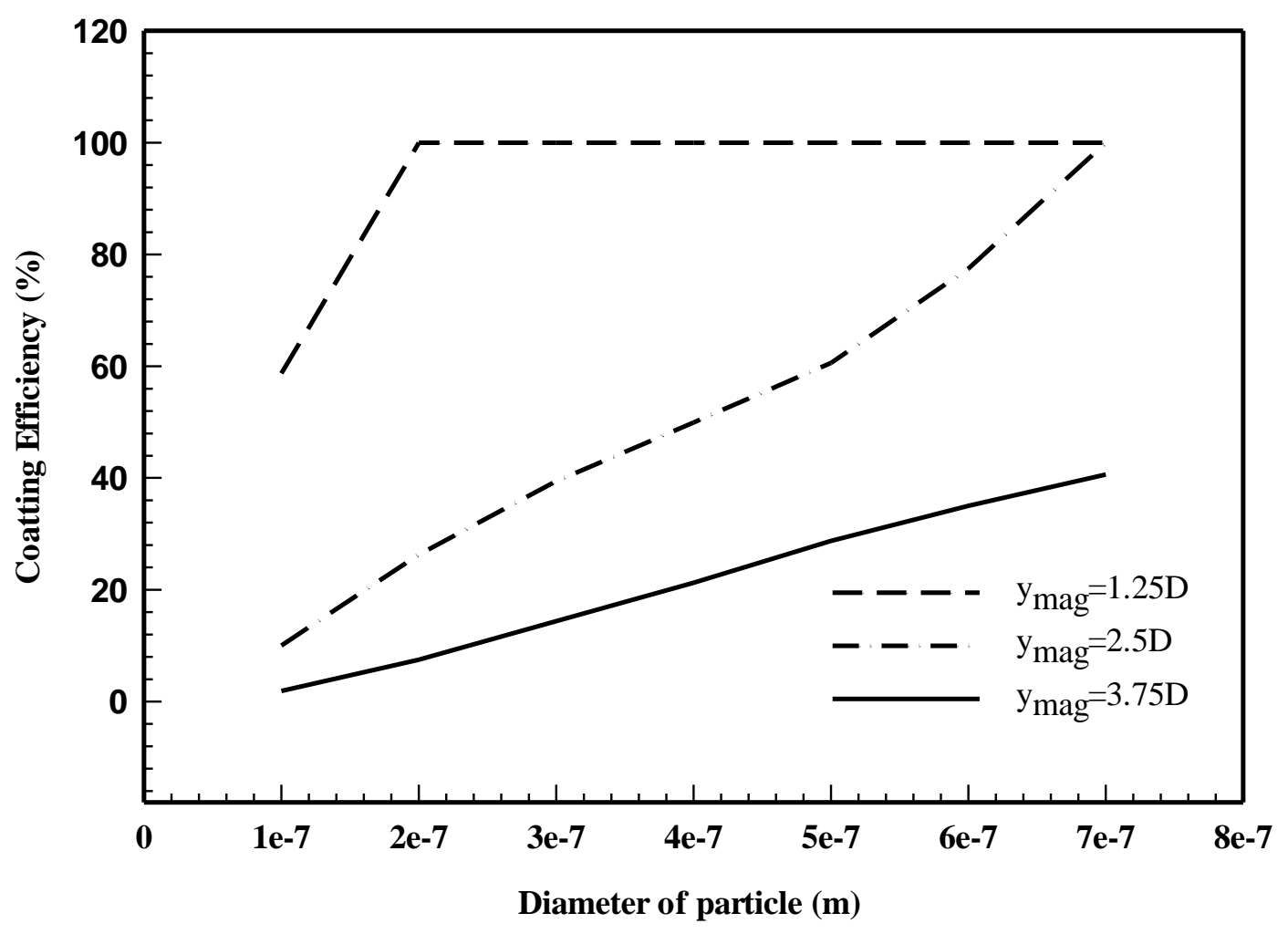

Fig. 10. Variations of coating efficiency with magnetic nanoparticles diameter for three wire positions at $\mathrm{Re}=100$ and $\mathrm{B}_{\max }=1.5$ Tesla. 
Table 1: Physical properties of magnetic nanofluid at $293 \mathrm{~K}$

\begin{tabular}{ccc}
\hline Properties & Water & $\mathrm{Fe}_{3} \mathrm{O}_{4}$ \\
\hline$\rho\left(\mathrm{kg} / \mathrm{m}^{3}\right)$ & 998 & 5200 \\
$\mu\left(N . \mathrm{s} / \mathrm{m}^{2}\right)$ & 0.001003 & - \\
\hline
\end{tabular}


Table 2: The effects of grid number on coating efficiency for $\operatorname{Re}=100, \mathrm{ymag}_{\mathrm{ma}}=2.5 \mathrm{~cm}$ and $\mathrm{dp}=10^{-7}$

\begin{tabular}{cccc}
\hline No. & Grid number & Coating efficiency $(\boldsymbol{\%})$ & Percentage differences \\
\hline 1 & $40 \times 250$ & 9.9290 & 1.7 \\
2 & $80 \times 500$ & 10.0978 & 0.9 \\
3 & $160 \times 1000$ & 10.1887 & 0.4 \\
4 & $320 \times 2000$ & 10.2294 & $-\cdots--$ \\
\hline
\end{tabular}

